\title{
Polymer-Based Micro Flow Sensor with Alternative Electronic Signal Interfaces for Low and High Flow Rates
}

\author{
Thomas Schönstein ${ }^{*}$ \\ Head Application Development, Innovative Sensor Technology IST USA Division, Las Vegas 89147, USA
}

Received: July 30, 2013 / Accepted: August 21, 2013 / Published: April 25, 2014

\begin{abstract}
The qualitative and quantitative assessment of gas flow has become increasingly relevant in the use of everyday systems. The micro flow sensor, developed by Innovative Sensor Technology AG (Switzerland), is by principle a calorimetric flow sensor produced as a micro system on a glass substrate by means of photolithography and glass etching technology. These structures are arranged as a platinum micro heater and sensor in a Wheatstone bridge. The subsequent etching process produces an exposed area of polyimide membrane that is only a few microns thick and includes the resistive sensor structure as the active area. In addition, the RTD (resistance temperature detector) technology included on the sensor allows for the implementation of a variety of electronic biasing and signal processing modes. Since the sensor can be powered and the bridge can be measured in both CTA (constant temperature anemometer) and calorimetric mode, new possibilities are presented for both low and high flow rates with regard to temperature compensation, self-calibration and self-monitoring.
\end{abstract}

Key words: Calorimetric flow sensor, pick \& place assembly, CTA-mode, low and high flow rates.

\section{Introduction}

The sensors are characterized by a very small time response and low power consumption, achieved through the principle of MEMs (Micro-Electro-Mechanical Systems) hot film anemometry. Despite an extremely thin membrane, the glass carrier produces a robust sensor and provides easy handling. The heater/sensor structure, in combination with the full bridge circuitry "on chip", ensures simple signal evaluation and offers the ability to detect the flow direction. The thin film sputtering and etching processes of IST AG (Innovative Sensor Technology AG) allow for low initial development costs for application-specific, custom sensor designs and support cost effective volume production. A wide range of sensor dimensions, resistance values, structural formations and mounting configurations are possible. As a result,

\footnotetext{
*Corresponding author: Thomas Schönstein, Dipl. Eng.. E-mail: thomas.schoenstein@ist-ag.com.
}

cost effective solutions including a sensor on $\mathrm{PCB}$ (printed circuit board) with electronic signal processing and flow channel can be easily developed in a timely manner.

\section{Sensor Advantages}

Compared with its silicon-based counterparts, the micro flow sensor (Fig. 6) from IST AG offers major advantages relating to dynamic measuring range and assembly of the sensing element into customer systems for design-in processes (Fig. 1). Due to the sensor's ability to operate in a combination of CTA (constant temperature anemometer) and calorimetric mode, the flow signal saturation can be shifted from $1 \mathrm{~m} / \mathrm{s}$ (only calorimetric mode) to $50 \mathrm{~m} / \mathrm{s}$ (both modes combined). These flow velocities translate to a volumetric flow range from $0.001 \mathrm{~L} / \mathrm{min}$ full scale to $10 \mathrm{~L} / \mathrm{min}$ full scale (depending on the flow channel cross-sectional area) with a possible resolution of $1 \%$ 
of the full scale value.

The response time of $10 \mathrm{~ms}$ and warm up time of $100 \mathrm{~ms}$ allows for time-based low power heater management. With the platinum technology of all resistive structures, a heater temperature regulation loop can be realized in order to maintain a constant temperature and to protect the sensor against damage from overheating. Especially during power down cycles, the resistive structures can be used to measure the gas temperature. As a result, it is possible to maintain active temperature compensation by tracking the heating power of the sensor's micro heater.

\section{Sensor Assembly}

The sensors can be produced economically and are well-suited for pick \& place assembly. It can be handled similar to an IC-die or SMD (surface mount device) component, assembled by standard pick \& place machines or by hand. Electronic connections to a PCB can be achieved through wire bonding or flip chip technology. The sensor can be assembled over a milled PCB channel or directly embedded into a PCB cavity (Fig. 1). The goal of IST's sensor/electronic interface development is to provide customers with an easy-to-use measurement concept for realizing their own proprietary solutions down to the component level of flow measurement technique.

\section{Calorimeter Mode}

The most common used measuring mode for micro machined flow sensors is the calorimetric mode. Here the temperature distribution along a sensor-heater-sensor-structure depending on gas flow is used to provide an electrical differential signal by thermal imbalance of upstream and downstream sensor elements (e.g. thermopiles) (Fig. 2).

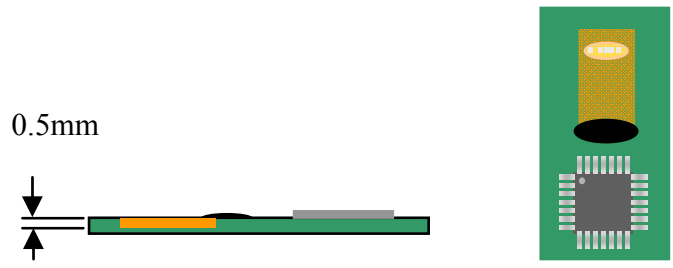

Fig. 1 Assembly example of micro flow sensor.

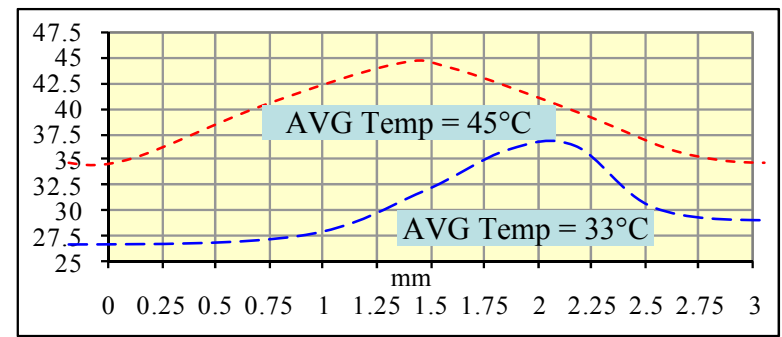

Fig. 2 Example of symmetrical and imbalanced temperature distribution on a $3 \mathrm{~mm}$ sensor area.

In case of a flow rate near by $200 \mathrm{~mL} / \mathrm{min}$, a temperature difference of some degree centigrade $\left({ }^{\circ} \mathrm{C}\right)$ between up- and down-stream sensor appears and causes a bridge signal of about $40-50 \mathrm{mV}$. Here, the user is faced with premature saturation effects, preventing high flow applications. This can be eliminated by using both flow measuring modes at one sensor structure (calorimetric and anemometric mode). The anemometric mode, especially the CTA-mode, is responsible for compensating the loss of heating power caused by gas flow. So an extended measuring range of calorimetric mode can be achieved. And additionally the output of the CTA can be used up to flow rates of $10 \mathrm{~L} / \mathrm{min}$ (Fig. 3).

\section{CTA-Mode}

The most popular anemometer is the CTA. It is function is well described by the King's Law:

$$
\begin{gathered}
P_{H}=I_{H}^{2} \cdot R_{H}=\left(A+B \cdot \vec{v}^{n}\right) \cdot \Delta T \\
n=0.3 \cdots 0.5
\end{gathered}
$$

By converting and simplifying this equation we obtain this formula:

$$
U=U_{0} \cdot \sqrt{1+k \cdot \vec{v}^{n}}
$$

where, $U$ : CTA-output; $U_{0}$ : free convection offset; $k$ : fluidic depend constant; $\vec{v}$ : fluid velocity.

Thus, the output is determined by an offset $\left(U_{0}\right)$, an offset dependent slope of the curve, and the fluidic dependent gain value $(k)$.

Here, $U_{0}$ represents the value of constant temperature difference ( $\Delta T$ ) between the heater and fluid. Strictly speaking, the controller of a CTA keeps the resistive structure at a constant temperature. So, 


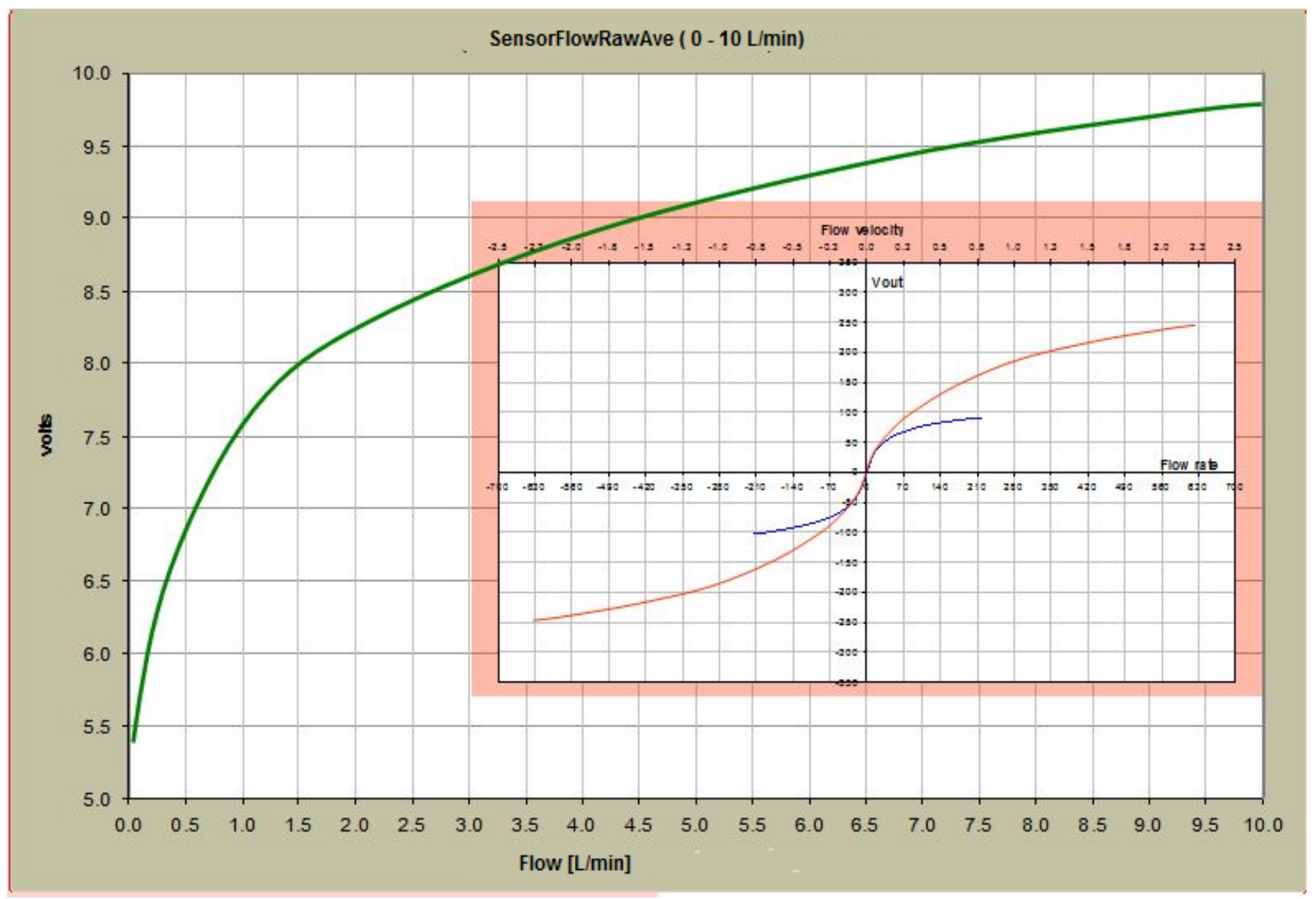

Fig. 3 Example of extended flow range by using CTA-output.

different passivation thicknesses and flow element surfaces impact the CTA characteristics, even a deviation in the heat transfer coefficient $(\alpha)$. The characteristics depend not only on sensor alignment/orientation and fluid type, but also on fluid temperature and sensor contamination (such as dust).

So the equation for a still fluid is:

$$
\begin{gathered}
\alpha_{0} \cdot A \cdot \Delta T \sim U_{0}^{2}\left(1+k \cdot \vec{v}^{n}\right) \\
\alpha_{0} \cdot A \cdot \Delta T \sim U_{0}^{2}
\end{gathered}
$$

That means each deviation of CTA-characteristic, caused by free convective parameters like $\alpha_{0}$ can be compensated by a $U_{0}$-adjustment at $\vec{v}=0$. In order to calibrate the $k$-value (fluidic-caused errors) more than one point is necessary. But, fluidic-caused errors mostly depend on mechanical alignment deviations of the sensor mounting. If mounting errors can be determined by module construction, a one point calibration provides good results for many applications.

\section{Chip Design and Circuit}

To get a high flow rate sensor circuit a microflow sensor has to be implemented into a CTA-bridge.

The complete 4-resistor sensor bridge acts as the heater of the CTA (Rs1, $\cdots$, Rs4). The outputs "Flow Dir" provide once the calorimetric mode and also the signal for flow direction.

4-resistor + Temp. sensor

Using as full bridge in a CTA<smiles>C=[Tl]</smiles>

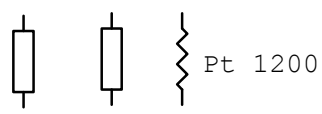

Flow direction sensitive

Fig. 4 Four resistors layout with temperature compensation. 

Interfaces for Low and High Flow Rates

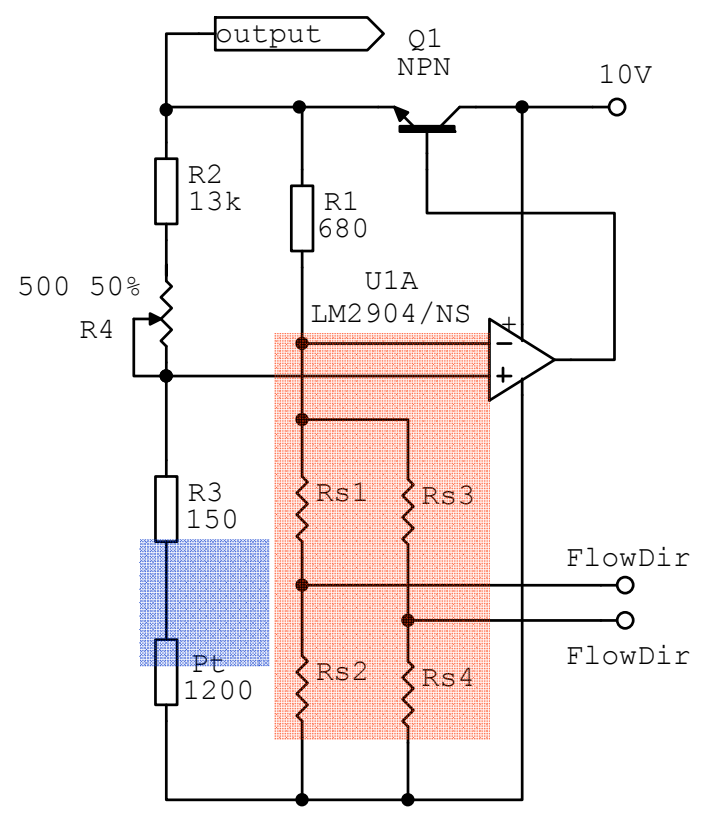

Fig. 5 Sensor layout and CTA circuit.

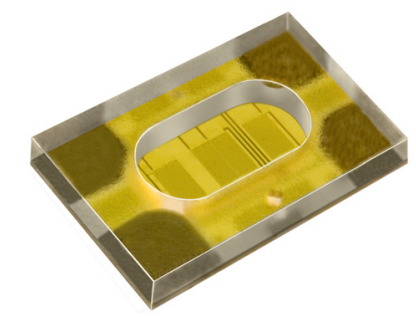

Fig. 6 Microflow sensor element $3 \mathrm{~mm} \times 5 \mathrm{~mm} \times 0.5 \mathrm{~mm}$.

\section{References}

[1] Brokmann, U.: Contribute to the Micro Structure of Photo-Imagable Glass by UV Radiation. Ilmenau: TU, 2005.

[2] Mrotzek, S.; Hülsenberg, D.; Hesse, A. Crystallization of a Glass in the Photo-Structured System $\mathrm{Li}_{2} \mathrm{O}-\mathrm{Al}_{2} \mathrm{O}_{3}-\mathrm{SiO}_{2}$. Ilmenau: TU.

[3] Technical Glass Works Ilmenau: Photo Structurable Glass for Micro-Engineering Applications. fuko_flyer.pdf, Ilmenau. 